\title{
Gravity wave analogs of black holes
}

\author{
Ralf Schützhold and William G. Unruh \\ Department of Physics and Astronomy, University of British Columbia, Vancouver, B.C., V6T $1 Z 1$ Canada \\ Electronic addresses: schuetz@physics.ubc.ca, unruh@physics.ubc.ca
}

(February 4, 2008)

\begin{abstract}
It is demonstrated that gravity waves of a flowing fluid in a shallow basin can be used to simulate phenomena around black holes in the laboratory. Since the speed of the gravity waves as well as their high-wavenumber dispersion (subluminal vs. superluminal) can be adjusted easily by varying the height of the fluid (and its surface tension) this scenario has certain advantages over the sonic and dielectric black hole analogs, for example, although its use in testing quantum effects is dubious. It can be used to investigate the various classical instabilities associated with black (and white) holes experimentally, including positive and negative norm mode mixing at horizons.

PACS: 04.70.-s, 47.90.+a, 92.60.Dj, 04.80.-y.
\end{abstract}

\section{INTRODUCTION}

One of the most fascinating predictions of Einstein's theory of general relativity is the potential existence of black holes - i.e. space-time regions from which nothing is able to escape. Perhaps no less interesting are their antonyms: white holes (nothing can penetrate). Both are described by solutions of the Einstein equations and are related to each other via time-inversion, see e.g. [1].

As it is well-known, these objects feature many novel properties: For example, for orbits sufficiently close to the horizon (i.e. for $r<3 M$ ) one observes [2] an inversion of the centrifugal acceleration.

Rotating black holes as described by the Kerr metric admit unstable modes under certain conditions, i.e. solutions of the wave equation growing in time without any bound, see e.g. [3, th. This phenomenon is related to the mechanism of superradiance [5] which allows one to extract energy from the rotation of the Kerr black hole, cf. [1].

White holes are unstable [6] to exponential build-up of energy on the white hole Cauchy horizon on the classical level, as well as on the quantum level [7] 9 .

The presence of both Cauchy and particle horizons (white and black hole horizons), such as in the interior of a Reissner-Nordström metric, can have further instabilities, see e.g. [10].

Another striking effect is the evaporation [11] of black holes due to quantum effects. This observation can be interpreted as a confirmation of their thermodynamical interpretation [12] relating purely geometrical quantities, such as surface gravity and surface area, to thermal properties, such as temperature and entropy.

Fortunately it seems unlikely that one can observe black holes in the laboratory (see, however, e.g. [13|). Analogs, which obey similar equations of motion to fields around a black hole raise the possibility of demonstrating some of the most unusual properties of black holes in the laboratory. This is the basic idea of the black and white hole analogs (Dumb holes) originally proposed by Unruh in Ref. [14. The sonic analogs established there are based on the observation that sound waves in flowing fluids are (under appropriate conditions) governed by the same wave equation as a scalar field in a curved spacetime. The acoustic horizon, which occurs if the velocity of the fluid exceeds the speed of sound within the liquid, acts on sound waves exactly as a black hole horizon does on, for example, scalar waves.

After the original proposal in Ref. 14] the sonic analogs have been the subject of several investigations, see e.g. [15 177. Although the kinematics of the waves propagating within the black and white hole analogs are governed by the same equation as those in a curved space-time, the dynamics of the effective metric itself are not described by the same laws as gravity (i.e. the Einstein equations) in general.

In this way the analogs allow one to separate the dynamical effects of gravity (following from the Einstein equations) from more general (kinematic) phenomena, cf. [19,20.

In addition to the sonic analogs there exist proposals for black or white hole analogs based on the propagation of light in dielectric media (instead of sound), see e.g. 20 24, and of other sorts of waves in for example liquid Helium 3, see e.g. [16]. These scenarios avoid some of the difficulties associated to the sonic analogs but can have other problems.

The challenge in making such analogs to horizons is in preparing a medium in which the waves are stopped from propagating out from some region. In the analogs where the flow of a medium is used to drag the waves at a velocity corresponding to the velocity of the waves, one requires a sufficiently low velocity that the experiment could be contemplated. The speed of sound depends on the equation of state $p=p(\varrho)$ only and therefore is hard to adjust by external parameters. In the case of the analogs based on light, the velocity of the (quasi) photons is determined by the effective permittivity and permeability of the medium, which can also be hard to

\footnotetext{
* There are, however, possibilities to reproduce the Einstein equations even in non-gravitational systems, see 18.
} 
manipulate, and especially hard to make a sufficiently low group and phase velocity of light (cf. 22, 24, 25]).

Consequently we were led to look for another kind of waves traveling at a velocity that can be controlled more easily. One promising candidate is gravity waves (surface waves) in a shallow basin filled with a liquid. As we shall see in the following, long gravity waves within a flowing fluid are also governed by the same wave equation as a scalar field in a curved space-time. In addition, the speed of the long gravity waves can be adjusted very easily by varying the depth of the basin. Furthermore the fluid flow in such a basin is easily manipulated. Because of the low velocity of these waves, quantum effects would not be observable, but many of the classical features of black holes (including the positive and negative norm mixing at the horizon which is closely related to the quantum evaporation effects) could be investigated. Furthermore, as we shall see, the dispersion relation of these waves at high wavenumbers can also be easily manipulated, allowing easy investigations of the effects of such changes on horizon effects.

\section{THE MODEL}

We shall begin with the simplest form of the model, in which we assume a shallow liquid over a flat, horizontal bottom. Furthermore, the forces on the liquid will be assumed to be such that they allow for a purely horizontal stationary flow profile resulting in a constant height (i.e. horizontal surface) of the liquid. Later we shall relax both of these assumptions. In addition, we shall assume that the liquid is viscosity free, incompressible and irrotational in its flow.

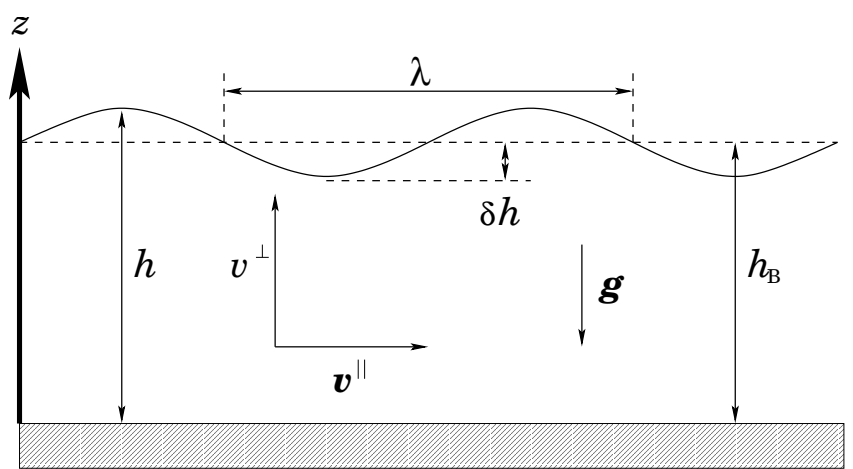

FIG. 1. Picture of a gravity wave in the basin and the relevant parameters. The assumed relation of the dimensions for long gravity waves $\delta h \ll h_{\mathrm{B}} \ll \lambda$ is not reproduced for the sake of conciseness.

In such a case the density of the liquid remains constant ( $\varrho=$ const) and in terms of its local velocity $\boldsymbol{v}$ the equation of continuity assumes the simple form

$$
\boldsymbol{\nabla} \cdot \boldsymbol{v}=0
$$

If we neglect the viscosity of the fluid its dynamics are governed by the non-linear Euler equations, see e.g. 226]

$$
\frac{d \boldsymbol{v}}{d t}=\dot{\boldsymbol{v}}+(\boldsymbol{v} \cdot \nabla) \boldsymbol{v}=-\frac{\boldsymbol{\nabla} p}{\varrho}+\boldsymbol{g}+\frac{\boldsymbol{f}}{\varrho},
$$

with $p$ denoting the pressure and $\boldsymbol{g}=-g \boldsymbol{e}_{z}$ the gravitational acceleration; and $f=-\varrho \boldsymbol{\nabla}_{\|} V^{\|}$is some horizontal force necessary to establish the stationary horizontal flow; cf. Fig. 1.

For an irrotational flow profile $\boldsymbol{\nabla} \times \boldsymbol{v}=0$ we may simplify the Euler equation (2) via $(\boldsymbol{v} \cdot \boldsymbol{\nabla}) \boldsymbol{v}=\boldsymbol{\nabla}\left(\boldsymbol{v}^{2}\right) / 2$ and introduce a velocity potential $\boldsymbol{v}=\boldsymbol{\nabla} \Phi$ arriving at the Bernoulli equation

$$
\dot{\Phi}+\frac{1}{2}(\nabla \Phi)^{2}=-\frac{p}{\varrho}-g z-V^{\|} .
$$

The boundary conditions are that the vertical velocity must be zero at the bottom of the tank, the pressure zero at the displaced surface, and change in the height of the fluid determined by the vertical velocity (cf. Fig. 1)

$$
v^{\perp}(z=0)=0, \quad v^{\perp}(z=h)=\frac{d h}{d t}=\dot{h}+(\boldsymbol{v} \cdot \nabla) h,
$$

and

$$
p(z=h)=0 .
$$

Now, let us consider perturbations $\delta \boldsymbol{v}$ to a background flow $\boldsymbol{v}_{\mathrm{B}}$ (which is assumed to be stationary, irrotational, and horizontal) corresponding to small vertical displacements $\delta h$ of the height of the fluid, $h$. The background flow will be assumed to obey

$$
\nabla_{\perp} \boldsymbol{v}_{\mathrm{B}}=0, \quad \boldsymbol{v}_{\mathrm{B}}=\boldsymbol{v}_{\mathrm{B}}^{\|} \leadsto \nabla_{\|} \cdot \boldsymbol{v}_{\mathrm{B}}=0,
$$

i.e., $h_{\mathrm{B}}=$ const, and

$$
\frac{1}{2} \boldsymbol{v}_{\mathrm{B}}^{2}=-\frac{p_{\mathrm{B}}}{\varrho}-g z-V^{\|},
$$

where $p_{\mathrm{B}}$ will be given by $g\left(h_{\mathrm{B}}-z\right)$.

We shall assume that the velocity perturbations are also irrotational, so that they are given by a potential, $\delta \Phi$. The perturbations of the Bernoulli equation are given by

$$
\dot{\delta \Phi}+\boldsymbol{v}_{\mathrm{B}}^{\|} \cdot \nabla_{\|} \delta \Phi=-\frac{\delta p}{\varrho} .
$$

The boundary condition (5) for the pressure together with $p_{\mathrm{B}}=g\left(h_{\mathrm{B}}-z\right)$ imply

$$
\delta p\left(z=h_{\mathrm{B}}\right)=g \varrho \delta h,
$$

and similarly for the vertical velocity

$$
\delta v^{\perp}\left(z=h_{\mathrm{B}}\right)=\dot{\delta h}+\left(\boldsymbol{v}_{\mathrm{B}}^{\|} \cdot \nabla_{\|}\right) \delta h,
$$

as well as $\delta v^{\perp}(z=0)=0$.

It is useful to expand the perturbation potential $\delta \Phi$ into a Taylor series 


$$
\delta \Phi(x, y, z)=\sum_{n=0}^{\infty} \frac{z^{n}}{n !} \delta \Phi_{(n)}(x, y)
$$

The boundary condition in Eq. (价 implies $\delta \Phi_{(1)}=0$. Another constraint arises from the equation of continuity (11)

$$
\nabla_{\|}^{2} \delta \Phi_{(0)}+\delta \Phi_{(2)}=0,
$$

and so on for larger values of $n$. We assume that the wavelength $\lambda$ of the perturbation is much longer than the depth $h_{\mathrm{B}}$. In this case the higher-order terms in the Taylor expansion (11) are suppressed by powers of $h_{\mathrm{B}} / \lambda \ll 1$ since we have $\nabla_{\|}^{2}=\mathcal{O}\left(1 / \lambda^{2}\right)$. Keeping only the two lowest (non-trivial) terms in Eq. (12) we find that

$$
\delta v^{\perp}\left(z=h_{\mathrm{B}}\right)=-h_{\mathrm{B}} \nabla_{\|}^{2} \delta \Phi_{(0)} .
$$

This enables us to combine Eqs. (8), (9), and (10)

$$
\left(\frac{\partial}{\partial t}+\boldsymbol{v}_{\mathrm{B}}^{\|} \cdot \boldsymbol{\nabla}_{\|}\right)^{2} \delta \Phi_{(0)}-g h_{\mathrm{B}} \boldsymbol{\nabla}_{\|}^{2} \delta \Phi_{(0)}=0 .
$$

This wave equation, however, equals the Klein-FockGordon (KFG) equation

$$
\square \delta \Phi_{(0)}=\frac{1}{\sqrt{-g}} \partial_{\mu}\left(\sqrt{-g} g^{\mu \nu} \partial_{\nu} \delta \Phi_{(0)}\right)=0,
$$

with the effective metric (remember $\boldsymbol{\nabla}_{\|} \cdot \boldsymbol{v}_{\mathrm{B}}^{\|}=0$ and $g h_{\mathrm{B}}=$ const)

$$
\mathfrak{g}_{\mathrm{eff}}^{\mu \nu}=\left(\begin{array}{cc}
1 & \boldsymbol{v}_{\mathrm{B}}^{\|} \\
\boldsymbol{v}_{\mathrm{B}}^{\|} & \boldsymbol{v}_{\mathrm{B}}^{\|} \otimes \boldsymbol{v}_{\mathrm{B}}^{\|}-g h_{\mathrm{B}} \mathbf{1}
\end{array}\right) .
$$

Except for replacing the velocity of the gravity waves $\sqrt{g h_{\mathrm{B}}}$ by the speed of sound it is exactly the same effective metric as for the sonic analogs. Calculating the inverse $\mathfrak{g}_{\mu \nu}^{\text {eff }}$ of the effective metric one obtains

$$
\mathfrak{g}_{00}^{\mathrm{eff}}=1-\left(\frac{\boldsymbol{v}_{\mathrm{B}}^{\|}}{\sqrt{g h_{\mathrm{B}}}}\right)^{2} .
$$

As one would expect, the condition of an ergosphere $\mathfrak{g}_{00}^{\text {eff }}=0$ is fulfilled where the velocity of the fluid $\boldsymbol{v}_{\mathrm{B}}^{\|}$ equals the speed of the gravity waves $\sqrt{g h_{\mathrm{B}}}$.

\section{ARBITRARY BOTTOM AND HEIGHT}

Let us now relax the previous assumptions that the bottom and the background flow surface are both flat and parallel. We introduce arbitrary coordinates on the bottom and define a vertical coordinate $z$ as orthogonal to the bottom of the container and geodesic. The spatial metric can always be cast into the form

$$
d \boldsymbol{r}^{2}=d z^{2}+\eta_{i j} d x^{i} d x^{j},
$$

where $i j$ go over values 1 and 2 (Einstein summation convention), and represent the coordinates within the bottom of the container. The equation of continuity (II) is now

$$
\partial_{i}\left(\sqrt{\eta} v^{i}\right)+\partial_{z}\left(\sqrt{\eta} v^{z}\right)=0,
$$

with $\eta=\operatorname{det}\left(\eta_{i j}\right)$ and, assuming irrotational flow, the Bernoulli equation (3) becomes

$$
\dot{\Phi}+\eta^{i j}\left(\partial_{i} \Phi\right)\left(\partial_{j} \Phi\right)+\left(\partial_{z} \Phi\right)^{2}=-\frac{p}{\varrho}-V\left(x^{i}, z\right),
$$

with

$$
v_{i}=\eta_{i j} v^{j}=\partial_{i} \Phi
$$

and

$$
v_{z}=v^{z}=\partial_{z} \Phi .
$$

Here the potential $V\left(x^{i}, z\right)$ already includes the gravitational acceleration - in contrast to the potential $V^{\|}$used in the previous Section.

The surface of the liquid, defined by $z=h\left(x^{i}\right)$, is where the pressure goes to zero and obeys, cf. Eq. (雨)

$$
\dot{h}+v^{i} \partial_{i} h=v^{z},
$$

where $v^{i}$ and $v^{z}$ are evaluated at the surface.

Let us now expand these expressions in powers of the vertical height $z$ above the bottom at $z=0$. The velocity potential $\Phi$, the metric $\eta^{i j}$, and the potential $V$ can be written as

$$
\begin{gathered}
\Phi\left(x^{i}, z\right)=\Phi_{(0)}\left(x^{i}\right)+\frac{z^{2}}{2} \Phi_{(2)}\left(x^{i}\right)+\mathcal{O}\left(z^{3}\right), \\
\eta^{i j}\left(x^{i}, z\right)=\eta_{(0)}^{i j}\left(x^{i}\right)+z \eta_{(1)}^{i j}\left(x^{i}\right)+\mathcal{O}\left(z^{2}\right),
\end{gathered}
$$

and

$$
V\left(x^{i}, z\right)=V_{(0)}\left(x^{i}\right)+z g_{z}\left(x^{i}\right)+\mathcal{O}\left(z^{2}\right),
$$

where we already have incorporated the boundary condition $v_{z}(z=0)=0$ and introduced the gravitational acceleration perpendicular to the bottom $g_{z}=V_{(1)}$. Similarly we obtain for the pressure $p$

$$
p=(h-z) p_{(1)}+\mathcal{O}\left([h-z]^{2}\right) .
$$

In analogy to the previous Section we assume the height $h$ of the fluid to be much smaller than the horizontal length scales on which the features of the flow profile (e.g. $\Phi, \eta^{i j}$, $V$, and $p$ ) change significantly - such as the wavelength $\lambda$. In this long-wavelength limit the higher-order terms of the above Taylor expansions are supressed by powers of $h / \lambda$ and thus can be neglected.

The continuity equation (1) enforces again

$$
\begin{aligned}
\frac{1}{\sqrt{\eta_{(0)}}} \partial_{i}\left(\sqrt{\eta_{(0)}} \eta_{(0)}^{i j} \partial_{j} \Phi_{(0)}\right)+\Phi_{(2)} & \equiv \\
\nabla_{\|}^{2} \Phi_{(0)}+\Phi_{(2)} & =0 .
\end{aligned}
$$


Evaluated at $z=h$ the equation (23) for the height in terms of the velocity reads

$$
\dot{h}+\eta^{i j}\left[\partial_{i} \Phi\left(x^{k}, h\right)\right] \partial_{j} h=\partial_{z} \Phi\left(x^{k}, h\right),
$$

which again to lowest order in $z=h$ becomes

$$
\dot{h}+\eta_{(0)}^{i j}\left(\partial_{i} \Phi_{(0)}\right) \partial_{j} h=-h \nabla_{\|}^{2} \Phi_{(0)}+\mathcal{O}\left(h^{2}\right),
$$

or, equivalently,

$$
\dot{h}+\frac{1}{\sqrt{\eta_{(0)}}} \partial_{i}\left(\sqrt{\eta_{(0)}} \eta_{(0)}^{i j} h \partial_{j} \Phi_{(0)}\right)=0,
$$

which can be interpreted as an effective conservation law $\dot{h}+\nabla_{\|} \cdot\left(h \boldsymbol{v}^{\|}\right)=0$.

The Bernoulli equation transforms into

$$
\begin{aligned}
\dot{\Phi}_{(0)} & +\frac{1}{2}\left(\eta_{(0)}^{i j}+z \eta_{(1)}^{i j}\right)\left(\partial_{i} \Phi_{(0)}\right)\left(\partial_{j} \Phi_{(0)}\right) \\
& =-\frac{p_{(1)}}{\varrho}(h-z)-V_{(0)}-z g_{z}+\mathcal{O}\left(h^{2}\right) .
\end{aligned}
$$

From the terms linear in $z$ we may infer

$$
\frac{p_{(1)}}{\varrho}=g_{z}+\frac{1}{2} \eta_{(1)}^{i j}\left(\partial_{i} \Phi_{(0)}\right)\left(\partial_{j} \Phi_{(0)}\right) .
$$

We can define an effective gravitational acceleration as

$$
\widetilde{g}=g_{z}+\frac{1}{2} \eta_{(1)}^{i j}\left(\partial_{i} \Phi_{(0)}\right)\left(\partial_{j} \Phi_{(0)}\right) .
$$

Note that the eigenvalues $\kappa$ of $\eta_{(1)}^{i j}$ with respect to $\eta_{(0)}^{i j}$

$$
\eta_{(1)}^{i j} x_{j}=\kappa \eta_{(0)}^{i j} x_{j},
$$

are just twice the inverse of the principle radii of curvature of the surface over which the fluid is flowing. Thus the extra terms in Eq. (33) just represent the vertical centrifugal forces on the fluid travel over this curved surface, and in all of our following investigations will be negligible.

Let us assume again that we have a stationary background flow which obeys these equations, and we are interested in perturbations around this flow. The perturbation equations then are (using $v_{\mathrm{B}}^{i}=\eta_{(0)}^{i j} \partial_{j} \Phi_{(0)}^{\mathrm{B}}$ at $z=0$ )

$$
\begin{array}{r}
\dot{\delta h}+\frac{1}{\sqrt{\eta_{(0)}}} \partial_{i}\left(\sqrt{\eta_{(0)}} v_{\mathrm{B}}^{i} \delta h\right)= \\
-\frac{1}{\sqrt{\eta_{(0)}}} \partial_{i}\left(\sqrt{\eta_{(0)}} \eta_{(0)}^{i j} h_{\mathrm{B}} \partial_{j} \delta \Phi_{(0)}\right)
\end{array}
$$

and

$$
\dot{\delta \Phi_{(0)}}+v_{\mathrm{B}}^{i} \partial_{i} \delta \Phi_{(0)}=-\widetilde{g} \delta h .
$$

We can combine these equations to get

$$
\begin{array}{r}
\left(\partial_{t} \frac{1}{\widetilde{g}} \partial_{t}+\partial_{i} \frac{v_{\mathrm{B}}^{i}}{\widetilde{g}} \partial_{t}+\partial_{t} \frac{v_{\mathrm{B}}^{i}}{\widetilde{g}} \partial_{i}+\frac{1}{\sqrt{\eta_{(0)}}} \partial_{i} \frac{v_{\mathrm{B}}^{i} v_{\mathrm{B}}^{j}}{\widetilde{g}} \sqrt{\eta_{(0)}} \partial_{j}\right. \\
\left.-\frac{1}{\sqrt{\eta_{(0)}}} \partial_{i} h_{\mathrm{B}} \sqrt{\eta_{(0)}} \eta_{(0)}^{i j} \partial_{j}\right) \delta \Phi_{(0)}=0 .
\end{array}
$$

This again is a KFG equation with a metric given by

$$
\mathfrak{g}_{\mathrm{eff}}^{\mu \nu}=\frac{1}{h_{\mathrm{B}}^{2}}\left(\begin{array}{cc}
1 & v_{\mathrm{B}}^{i} \\
v_{\mathrm{B}}^{j} & v_{\mathrm{B}}^{i} v_{\mathrm{B}}^{j}-\widetilde{g} h_{\mathrm{B}} \eta_{(0)}^{i j}
\end{array}\right),
$$

where both $\widetilde{g}$ and $h_{\mathrm{B}}$ can depend on the coordinates $x^{k}$. We can thus sculpt the effective metric within which these waves flow both by altering the velocity of the background flow, by changing $\widetilde{g}$ from place to place (primarily by sloping the bottom of the tank), or by altering the height of the background flow. (Of course the background $h_{\mathrm{B}}$ is determined by the background flow, $\widetilde{g}$ and the potential $V$.) If one has only the gravitational field as a force on the fluid, the slope of the bottom can be used to generate a potential $V$, and also, with more severe slopes, to change the value of $\widetilde{g}$ from place to place.

The ergoregion is defined as the zone where, in order to be travelling at less than the velocity of the wave in the rest frame of the fluid, one cannot be standing still in the lab frame. This is the region where the velocity of the fluid is higher than the local velocity of the wave, $\sqrt{\widetilde{g} h_{\mathrm{B}}}$.

If we assume that the bottom is flat (so that $\eta^{i j}=\delta^{i j}$ ), and that the flow is driven by changes in $h_{\mathrm{B}}$, we have

$$
\frac{1}{2} v_{\mathrm{B}}^{2}+g h_{\mathrm{B}}=\text { const . }
$$

But $\sqrt{g h_{\mathrm{B}}}$ is the speed of the gravity waves. Far from the ergosphere, the velocity $v_{\mathrm{B}}$ is small, so the we have const $=g h_{\infty}$. Thus at the erogosphere, where the velocity of the fluid is the velocity of the waves, we have

$$
h=\frac{2}{3} h_{\infty} .
$$

Similarly, if we assume that we have a sloping bottom designed so that the fluid maintains a constant height $h_{\mathrm{B}}$ throughout, we would again obtain that the ergosphere should be at a point such that $V\left(x^{i}\right)-V_{\infty}=\widetilde{g} h_{\mathrm{B}} / 2$. If this potential arises purely from the gravitational potential due to the slope of the bottom, we must have that the bottom would have to be at a height of $h / 2$ lower than at infinity. I.e., it does not take much of slope to the bottom to create the conditions necessary for an analog black hole ergosphere to form.

We note that in the above, we have only kept terms to lowest order in $z$ or $h$. The validity of this approximation is essentially that all horizontal derivatives have scales which are much larger than $h$, the height of the fluid. Furthermore, the condition that we need only retain the lowest order in the metric $\eta^{i j}$ is also that the curvature of the bottom of the tank be on scales which are long with respect to $h$. I.e., we are in a "shallow water", long wavelength approximation in these derivations. 


\section{IRROTATIONAL BACKGROUND FLOW}

We can use the equations of motion of the fluid to derive the most general rotationally symmetric and locally irrotational backgound flow profile. Let us assume that the bottom of the tank is defined by the relation

$$
Z=f(R)
$$

in the usual cylindrical coordinates $(Z, R, \varphi)$, where $f$ denotes some moderately curved function. Switching to the adapted coordinates $(z, r, \varphi)$ described in the previous Section $(Z=f(R) \leftrightarrow z=0 ; r=R)$ gives the spatial metric

$$
\begin{aligned}
d \boldsymbol{r}^{2}=d z^{2} & +d r^{2}\left(1+f^{\prime}(r)^{2}\right)\left(1-z \frac{f^{\prime \prime}(r)}{\sqrt{\left(1+f^{\prime}(r)^{2}\right)^{3}}}\right)^{2} \\
& +d \varphi^{2}\left(r-z \frac{f^{\prime}(r)}{\sqrt{1+f^{\prime}(r)^{2}}}\right)^{2}
\end{aligned}
$$

for which the lowest order metric is

$$
d \boldsymbol{r}_{(0)}^{2}=d z^{2}+\left(1+f^{\prime}(r)^{2}\right) d r^{2}+r^{2} d \varphi^{2}
$$

To lowest order in $h$ the flow equations are the effective continuity equation (30)

$$
\partial_{r}\left(\sqrt{1+f^{\prime}(r)^{2}} r h v^{r}\right)=0
$$

the condition for a locally irrotational flow

$$
v^{\varphi}=\frac{L}{r^{2}}
$$

with $L$ being some constant related to the angular momentum, and finally the Bernoulli equation

$$
\begin{gathered}
\left(1+f^{\prime}(r)^{2}\right)\left(v^{r}\right)^{2}+r^{2}\left(v^{\varphi}\right)^{2}= \\
-g \frac{h-h_{\infty}}{\sqrt{1+f^{\prime}(r)^{2}}}-g f(r),
\end{gathered}
$$

where we have neglected the "centrifugal" term in Eq. (33) as it will be very small for our situation. These give

$$
v^{r}=\frac{C h_{\infty}}{r h \sqrt{1+f^{\prime}(r)^{2}}},
$$

and

$$
\frac{1}{2}\left(\frac{C^{2} h_{\infty}^{2}}{h^{2} r^{2}}+\frac{L^{2}}{r^{2}}\right)=-g \frac{h-h_{\infty}}{\sqrt{1+f^{\prime}(r)^{2}}}-g f(r) .
$$

Thus, we either need $h$ to change as a function of $r$, or we need a non-trivial $f(r)$. Choosing $f(r)=-F / r^{2}$ allows a consistant solution with contant height $h=h_{\infty}$ for the fluid as long as $F$ is given by $F=\left(C^{2}+L^{2}\right) / g$. The effective metric for the fluid is then of the form

$$
\begin{aligned}
d s_{\mathrm{eff}}^{2}= & \frac{h_{\infty}}{\widetilde{g}}\left(\widetilde{g} h_{\infty}-\frac{C^{2}+L^{2}}{r^{2}}\right) d t^{2} \\
& +2 \frac{h_{\infty}}{\widetilde{g}}\left(\sqrt{1+f^{\prime}(r)^{2}} \frac{C}{r} d t d r+L d t d \varphi\right) \\
& -\frac{h_{\infty}}{\widetilde{g}}\left(\left[1+f^{\prime}(r)^{2}\right] d r^{2}+r^{2} d \varphi^{2}\right)
\end{aligned}
$$

with $\widetilde{g}=g_{z}=g / \sqrt{1+f^{\prime}(r)^{2}}$ denoting the effective gravitational acceleration.

In summary the analogy to a curved space-time and the concept of an effective metric can still be applied in the case of non-horizontal flow provided that the local variation of the height of the fluid and the slope of its bottom are sufficiently small. Nevertheless, the global changes may well be significant.

A variation of $h$ and $g_{z}$, the component of the force perpendicular to the bottom, does in general also entail a change of the local velocity $\sqrt{g_{z} h}$ of the gravity waves. Such a spatial dependence may lead to further interesting effects: In analogy to optics one may introduce an effective index of refraction which then also acquires a non-negligible gradient. In such a situation the gravity waves may be scattered by this gradient or even the phenomenon of total reflection could occur. As we shall see later in Section $\mathrm{X}$, this mechanism may be one ingredient for generating an instability.

\section{SURFACE TENSION}

So far we have considered ideal fluids without any internal forces. However, if we take the surface tension of the liquid into account, the pressure at its surface no longer vanishes. Accordingly, the upper boundary condition (5) for the pressure is modified to

$$
p(z=h)=-\alpha \nabla_{\|}^{2} h,
$$

where $\alpha$ denotes the fluid's surface-tension coefficient and $\nabla_{\|}^{2} h$ is the curvature of its surface in the linear approximation. Consequently we obtain

$$
\delta p\left(h_{\mathrm{B}}\right)=\varrho g \delta h-\alpha \boldsymbol{\nabla}_{\|}^{2} \delta h,
$$

instead of Eq. (9). This results in a extra term in the velocity perturbation equation (8)

$$
\dot{\delta \Phi}+\boldsymbol{v}_{\mathrm{B}}^{\|} \cdot \nabla_{\|} \delta \Phi=-g \delta h+\frac{\alpha}{\varrho} \nabla_{\|}^{2} \delta h .
$$

As we shall see below, the effects of surface tension become relevant for small wavelengths only. In this limit we may neglect the variation of the background flow $\boldsymbol{\nabla}_{\|} \otimes \boldsymbol{v}_{\mathrm{B}}^{\|} \approx 0$ and obtain a modified wave equation

$$
\left(\frac{\partial}{\partial t}+\boldsymbol{v}_{\mathrm{B}}^{\|} \cdot \nabla_{\|}\right)^{2} \delta \Phi=g h_{\mathrm{B}} \nabla_{\|}^{2} \delta \Phi-\frac{\alpha h_{\mathrm{B}}}{\varrho} \nabla_{\|}^{4} \delta \Phi .
$$


In terms of the capillary constant $a^{2}=\alpha /(\varrho g)$ and the velocity of the unperturbed gravity waves $c_{\mathrm{B}}^{2}=g h_{\mathrm{B}}$ this wave equation results in the following dispersion relation

$$
\left(\omega+\boldsymbol{v}_{\mathrm{B}}^{\|} \cdot \boldsymbol{k}\right)^{2}=c_{\mathrm{B}}^{2}\left(\boldsymbol{k}^{2}+a^{2} \boldsymbol{k}^{4}\right) .
$$

Therefore the incorporation of the effects of surface tension leads to a "superluminal" dispersion relation (in the terminology of [27]) since (for $\boldsymbol{v}_{\mathrm{B}}^{\|}=0$ ) the group velocity $d \omega / d k$ as well as the phase velocity $\omega / k$ exceed $c_{\mathrm{B}}$ for large wavenumbers $k$.

However, we should bear in mind that the above calculations are still based on the assumption of long gravity waves $\lambda \gg h_{\mathrm{B}}$. For short gravity waves $\lambda \ll h_{\mathrm{B}}$, on the other hand, the dispersion relation reads (for $\boldsymbol{v}_{\mathrm{B}}^{\|}=0$, cf. [26])

$$
\omega^{2}=g k\left(1+a^{2} k^{2}\right) .
$$

Hence we can use the ratio $a / h_{\mathrm{B}}$ in order to alter the dispersion relation for large wavenumbers $k$ : For $a \gg h_{\mathrm{B}}$ the capillary waves dominate before the wavelength becomes smaller than the height, and we have a superluminal dispersion relation, whereas for $a \ll h_{\mathrm{B}}$ the short gravity waves dominate before the surface tension becomes important, and thus one initially has a subluminal dispersion relation, before the capillary waves finally take over at very short wavelengths.

For example, for mercury the surface tension coefficient $\alpha$ is about $\alpha \approx 0.46 \mathrm{~N} \mathrm{~m}^{-1}$ at room temperature $293 \mathrm{~K}$ and hence its capillary constant $a \approx 1.9 \mathrm{~mm}$. For water at $293 \mathrm{~K}$ we have $\alpha \approx 0.0725 \mathrm{~N} \mathrm{~m}^{-1}$ and hence $a \approx 2.7 \mathrm{~mm}$. This quantity can easily be manipulated by changing the temperature, adding surfactants, or by changing the fluid used.

\section{VISCOSITY}

The dynamics of a viscous but still incompressible fluid are governed by the Navier-Stokes equations

$$
\frac{d \boldsymbol{v}}{d t}=\dot{\boldsymbol{v}}+(\boldsymbol{v} \cdot \boldsymbol{\nabla}) \boldsymbol{v}=-\frac{\boldsymbol{\nabla} p}{\varrho}+\boldsymbol{g}+\nu \boldsymbol{\nabla}^{2} \boldsymbol{v},
$$

where $\varrho \nu$ denotes the dynamic viscosity of the liquid and $\nu$ its kinematic viscosity.

The boundary conditions have to be modified as well. Instead of Eq. (5) we have now

$$
p(z=h)=2 \varrho \nu \partial_{z} v_{z}(z=h),
$$

and there are two additional restrictions on $\boldsymbol{v}_{\|}$

$$
\partial_{z} \boldsymbol{v}_{\|}(z=h)=-\nabla_{\|} v_{z}(z=h),
$$

and

$$
\boldsymbol{v}_{\|}(z=0)=0 \text {. }
$$

Let us investigate the effects of a finite but small viscosity on the wave propagation - where we restrict our examination to the case of a vanishing background flow $\boldsymbol{v}_{\mathrm{B}}=0$ for simplicity and employ the plane-wave ansatz with a frequency $\omega$ and a wavenumber $\boldsymbol{k}$.

For an incompressible fluid the divergence of the linearized Eq. (56) yields (for $\boldsymbol{v}_{\mathrm{B}}=0$ )

$$
\nabla^{2} \delta p=\left(\partial_{z}^{2}-\boldsymbol{k}^{2}\right) \delta p=0,
$$

which has the solution $\delta p=A \cosh (k z)+B \sinh (k z)$ with $k=|\boldsymbol{k}|$.

In the long-wavelength limit $k h_{\mathrm{B}} \ll 1$ we may approximate $\sinh (k z) \approx k z$ and $\cosh (k z) \approx 1+(k z)^{2} / 2$. Inserting the resulting expression back into the Navier-Stokes equations (56) yields

$$
\left(\partial_{z}^{2}-k^{2}-i \frac{\omega}{\nu}\right) \delta v_{z}=\frac{A k^{2} z+B k}{\varrho \nu} .
$$

Defining $\widetilde{k}$ via $\widetilde{k}^{2}=k^{2}+i \omega / \nu$ and $\Re(\widetilde{k})>0$ the general solution of this equation can be written as

$$
\delta v_{z}=-\frac{A k^{2} z+B k}{\varrho \nu \widetilde{k}^{2}}+C e^{+\widetilde{k} z}+D e^{-\widetilde{k} z} .
$$

In addition to the long-wavelength limit $\lambda \gg h_{\mathrm{B}}$ we assume the viscosity $\nu$ to be very small $\nu \ll \omega h_{\mathrm{B}}^{2}$. In this case the exponentials $\exp ( \pm \widetilde{k} z)$ are very rapidly varying functions - which can be used to simplify the analysis.

Combining the equation of continuity $\partial_{z} \delta v_{z}+i \boldsymbol{k} \cdot \delta \boldsymbol{v}_{\|}=$ 0 with the velocity boundary condition in Eq. (58) one obtains $\left(\partial_{z}^{2}+k^{2}\right) \delta v_{z}\left(z=h_{\mathrm{B}}\right)=0$. In view of $|k| \gg k$ and $\widetilde{k} h_{\mathrm{B}} \gg 1$ this implies that $C$ is extremely small $C \propto$ $\exp \left(-\widetilde{k} h_{\mathrm{B}}\right) / \widetilde{k}^{2}$ and thus can be neglected.

On the other hand, from $\delta v_{z}(z=0)=0$ we obtain $D=B k /\left(\varrho \nu \widetilde{k}^{2}\right)$. Therefore, the term $D \exp (-\widetilde{k} z)$ in Eq. (62) is relevant in a very thin boundary layer of order $\sqrt{\nu / \omega}$ over the bottom only, cf. [26].

In analogy to Eq. (9) we may linearize the boundary condition for the pressure in Eq. (57) which fixes the integration constant $A$.

The remaining condition $\boldsymbol{v}_{\|}(z=0)=0$, i.e. $\partial_{z} \delta v_{z}(z=$ $0)=0$, can be used to eliminate $D$ and hence $B$. As one might expect, the solution for $\delta v_{z}$ in the presence of a small viscosity displays only slight deviations $(B$ is of order $\sqrt{\nu}$ ) from the linear profile $\delta v_{z} \propto z$ used in the previous Sections - as long as one is well above the aforementioned boundary layer.

Finally, Eq. (蛋), i.e. $\delta v_{z}\left(z=h_{\mathrm{B}}\right)=i \omega \delta h$, enables us to derive the dispersion relation

$$
\omega^{2}=g h_{\mathrm{B}} k^{2}-g k^{2} \sqrt{\frac{\nu}{i \omega}}+\mathcal{O}(\nu) .
$$

Here one can read off the characteristic damping time $\tau$ after which the viscosity effects become significant 


$$
\tau \sim \frac{h_{\mathrm{B}}}{\sqrt{\nu \omega}} .
$$

One observes that high frequencies are damped faster. This tendency becomes much stronger in the régime of short gravity waves where

$$
\tau \sim \frac{1}{\nu k^{2}} \sim \frac{g^{2}}{\nu \omega^{4}}
$$

holds, see e.g. 26. As a result, the at a first glance undesirable effects of viscosity can be utilized to damp out potential high-frequency noise and so single out the interesting (medium-wavenumber) instabilities by tuning $\nu$.

For example, water at room temperature has a kinematic viscosity of $\nu \approx 1 \mathrm{~mm}^{2} \mathrm{~s}^{-1}$. Assuming a height $h_{\mathrm{B}}=10 \mathrm{~cm}$ and a frequency $\omega=1 \mathrm{~Hz}$ we infer from Eq. (64) a damping time of the order of one minute - which still seems to be sufficient.

The kinematic effect of the friction within the liquid could be diminished by selecting a fluid with a relatively high density $\varrho$ (e.g. mercury in comparison with its inner viscosity. For mercury at room temperature $\nu$ is given by $\nu \approx 0.12 \mathrm{~mm}^{2} \mathrm{~s}^{-1}$.

However, there is also another problem induced by the finite viscosity: the ansatz for the $z$-independent background flow profile used in the previous Sections is not appropriate anymore since the fluid sticks to the bottom of the basin, cf. Eq. (59).

One way to solve this problem is to move the bottom so that its velocity is the same as that of the fluid at least in the interesting region, for example near the horizon. (One might also imagine manipulating the fluid near the bottom and thereby effectively simulating this motion.) After transforming into its rest frame the above calculation demonstrates that the solutions derived in the previous Sections are still a very good approximation.

Without moving the bottom the boundary condition in Eq. (59) enforces a significant $z$-dependence of the background flow profile. For instance a constant horizontal force (needed for maintaining the stationary flow) implies a parabolic flow profile $v_{\mathrm{B}}^{z} \propto z\left(2 h_{\mathrm{B}}-z\right)$. Unfortunately, it is not possible to cast the full wave-equation into an as tractable form as in Eq. (14) allowing for the identification of an effective metric in this situation (this problem is currently under investigation). The main obstacle is that the flow is longer irrotational.

Nevertheless, for other scenarios one might be able to overcome this difficulty. If one injects a nearly $z$ independent stationary inflow (driven by a turbine, for

\footnotetext{
$\dagger$ The use of a fluid like mercury has just another advantage since one would be able to detect the gravity waves very easily via considering the reflection at the fluid's surface in this case.
}

example) on one side of the basin, then the flow will basically remain irrotational throughout the basin - outside a thin Prandtl boundary layer at the bottom, cf. [26].

In view of the relatively large velocities involved and the supposedly small viscosity this layer may well be turbulent. However, by an appropriate preparation of the bottom's surface (e.g. dolphin skin effect) the indued drag can be diminished.

Since the properties of the flow outside the thin boundary layer are nearly the same as in the case without viscosity and in view of the remarks after Eq. (62) one would expect that the basic properties of the gravity waves as discussed in the previous Sections are not drastically affected by a small internal friction in this case.

Beside the scenarios described above there is also another - more exotic - solution for the viscosity problem conceivable: a superfluid does not stick to the boundary and its vorticity is quantized. But the necessity of using gravity waves in say liquid Helium makes the experimental realization far more difficult than is desirable.

\section{ENERGY}

There are two different concepts of a metric in our model: Firstly, the Minkowski metric determining the length- and time-scales within our laboratory; and, secondly, the effective metric - which is experienced by the gravity waves only.

These two concepts lead to two distinct notions of energy: For time-independent external forces (and inviscid fluids) the Noether theorem demands the conservation of the total energy of the flow. In addition, assuming a stationary background flow profile, we may find a conserved energy associated to the gravity waves:

At the boundary $\partial G$ of the basin, the normal component of the velocity $\boldsymbol{v} \cdot \boldsymbol{n}$ has to vanish. In terms of the velocity potential $\Phi$ this corresponds to Neumann boundary conditions

$$
\left.\boldsymbol{v} \cdot \boldsymbol{n}\right|_{\partial G}=\left.0 \leadsto \boldsymbol{n} \cdot \nabla \Phi\right|_{\partial G}=0 .
$$

This enables us to accomplish a spatial integration by parts and in complete analogy to the $2+1$ dimensional curved space-time one may derive a conserved energy

$$
E=\int d \Sigma_{\mu} T^{\mu \nu} \xi_{\nu}=\int d^{2} r T_{0}^{0}
$$

where $\xi^{\mu}=\partial / \partial t$ denotes the Killing vector associated to the physical laboratory time and $\Sigma_{\mu}$ the spatial hypersurface. For the effective metric in Eq. (16) we obtain the energy density

$$
T_{0}^{0}=\frac{1}{2}\left[\left(\frac{\partial \phi}{\partial t}\right)^{2}+g h_{\mathrm{B}}\left(\nabla_{\|} \phi\right)^{2}-\left(\boldsymbol{v}_{\mathrm{B}}^{\|} \cdot \nabla_{\|} \phi\right)^{2}\right]
$$

of the perturbations $\phi=\delta \Phi_{(0)}$, i.e. gravity waves (see also [28). 
We observe that this energy density contains negative parts inside the ergoregion $\left|\boldsymbol{v}_{\mathrm{B}}^{\|}\right|>\sqrt{g h_{\mathrm{B}}}$. This observation points to the possibility of an instability associated to the ergoregion. (A positive definite conserved energy density would prove stability.) The total energy of the fluid including the background flow is of course always positive.

Note that the energy conservation law derived above is violated if $G$ has a hole, such as at the drain, etc. This problem, however, arises for real black holes as well.

\section{NON-ROTATING BLACK HOLE}

If we neglect the small slope $f^{\prime}(r) \ll 1$ of the bottom (cf. the remarks at the end of Section III) in Eq. (49) the constant quantities $g$ and $h_{\mathrm{B}}$ can be absorbed by a simple rescaling and we arrive at

$$
\begin{aligned}
d s_{\mathrm{eff}}^{2}= & \left(g h_{\infty}-\frac{C^{2}+L^{2}}{r^{2}}\right) d t^{2}+2 \frac{C}{r} d t d r+2 L d t d \varphi \\
& -d r^{2}-r^{2} d \varphi^{2} .
\end{aligned}
$$

If we take $L=0$, we exactly recover a PainlevéGullstrand-Lemaître (PGL) type metric 29]

$d s_{\mathrm{eff}}^{2}=\left[c_{\mathrm{B}}^{2}-w^{2}(r)\right] d t^{2}+2 w(r) d t d r-d r^{2}-r^{2} d \varphi^{2}$,

with $c_{\mathrm{B}}^{2}=g h_{\infty}$ and $w(r)=\sqrt{1+f^{\prime}(r)^{2}} v^{r}=C / r$. As it is well-known, by means of the singular coordinate transformation

$$
d t \rightarrow d \tilde{t}=d t+d r \frac{w(r)}{c_{\mathrm{B}}^{2}-w^{2}(r)},
$$

the stationary PGL metric can be cast into the static Schwarzschild form

$$
d s_{\mathrm{eff}}^{2}=\left(c_{\mathrm{B}}^{2}-w^{2}\right) d \tilde{t}^{2}-\frac{c_{\mathrm{B}}^{2}}{c_{\mathrm{B}}^{2}-w^{2}} d r^{2}-r^{2} d \varphi^{2} .
$$

Obviously the horizon occurs when $w^{2}=c_{\mathrm{B}}^{2}=g h_{\mathrm{B}}$, i.e. when the velocity of the (radially) flowing fluid exceeds the speed of the (long) gravity waves $\sqrt{g h_{\mathrm{B}}}$. An inward flowing liquid $w<0$ simulates a black hole whereas an outward flow $w>0$ evidently corresponds to a white hole. The black hole branch can be used to observe the inversion of the centrifugal acceleration [2] mentioned in the Introduction and, of course, the trapping of the waves inside the horizon $w^{2}=c_{\mathrm{B}}^{2}$.

\section{WHITE HOLE}

The white hole branch $w>0$ of Eq. (70) offers another interesting phenomenon: As demonstrated in Ref. [6], all incident waves pile up at the horizon (since they cannot penetrate) and get arbitrarily blue-shifted there - if one neglects the change in dispersion relation, and thus group velocity, at high wavenumbers. In our model, however, the blue-shifted waves eventually leave the regime of the long gravity waves. The subsequent behavior depends on the character of the dispersion relation, in particular whether it is subluminal or superluminal. In the latter case the incident waves will eventually penetrate the horizon, once their wavelength has become sufficiently short to alter the group velocity to one larger than the (low frequency) velocity of the waves. In the former case, the short wavelengths will be swept out by the fluid which is now flowing faster at the horizon than the group velocity. These features associated with white holes may thus be observed experimentally for these gravity wave analogs. Furthermore, since the dispersion relation can be adjusted by varying the depth and the surface tension of the liquid, one can study the effect over a wide range of physical situations.

\section{ROTATING BLACK HOLE}

Stationary flow profiles containing a component in $\varphi$ direction (vortex solutions with non-zero $L$ ) can be used to model rotating (Kerr) black holes. With a further rescaling and a redefinition of the constants $C$ and $L$ we may absorb the speed of the gravity waves $g h_{\infty}$ in Eq. (69) completely and then the corresponding effective metric assumes the following form

$$
\begin{aligned}
d s_{\mathrm{eff}}^{2}= & \left(1-\frac{C^{2}+L^{2}}{r^{2}}\right) d t^{2}+2 \frac{C}{r} d t d r+2 L d t d \varphi \\
& -d r^{2}-r^{2} d \varphi^{2} .
\end{aligned}
$$

The space-time structure of a Kerr black hole is more complicated than the Schwarzschild geometry for there is a difference between the static limit (or the ergosphere, see e.g. [1]) and the horizon: The static limit $\mathfrak{g}_{00}=0$ denotes the region beyond which no particle can remain at rest. This, as mentioned above, corresponds to the surface where the velocity of the fluid equals the velocity of the waves, i.e. $r^{2}=C^{2}+L^{2}$. The horizon is the "point of no return", and for an axially symmetric flow, corresponds to the surface where the radial flow velocity equals the velocity of the waves $r^{2}=C^{2}$.

The region between these two critical points, in both Kerr space-time and this model, is called the ergoregion and allows for the occurrence of the superradiant modes.

According to Eq. (68) the energy density may become negative inside the ergoregion $r^{2}<C^{2}+L^{2}$. As already anticipated in Section VII, this observation can be interpreted as an indicator of instability. Indeed, in complete analogy with the Kerr black hole this analog should exhibits the phenomenon of superradiance: An incident wave with non-vanishing angular momentum scatters from the region around the black hole (analog) - i.e. the vortex - and the amplitude of the reflected wave is 
larger than that of the ingoing wave. The necessary energy is extracted from the rotational energy of the background.

Since the metric in Eq. (73) possesses two independent Killing vectors, $\partial / \partial t$ and $\partial / \partial \varphi$ we may find a complete set of solutions of the wave (KFG) equation

$$
\begin{array}{r}
\left(\partial_{t}^{2}+2 \frac{C}{r} \partial_{t} \partial_{r}+2 \frac{L}{r^{2}} \partial_{t} \partial_{\varphi}+\frac{C L}{r^{3}} \partial_{r} \partial_{\varphi}+\frac{1}{r} \partial_{r} \frac{C L}{r^{2}} \partial_{\varphi}\right. \\
\left.+\frac{1}{r} \partial_{r}\left(\frac{C^{2}}{r^{2}}-1\right) r \partial_{r}+\left(\frac{L^{2}}{r^{4}}-\frac{1}{r^{2}}\right) \partial_{\varphi}^{2}\right) \phi=0,
\end{array}
$$

by the following separation ansatz

$$
\phi(t, r, \varphi)=\exp \{-i \omega t+i m \varphi\} \phi_{\omega m}(r) .
$$

Note that an analogous separation ansatz in $t, \vartheta, \varphi$ is even possible in the real $3+1$ dimensional Kerr metric - which is a less trivial statement.

The remaining function $\phi_{\omega m}(r)$ obeys a second-order ordinary differential equation. In terms of the ReggeWheeler tortoise coordinate defined by

$$
d r_{*}=\frac{r^{2}}{r^{2}-C^{2}} d r
$$

the KFG equation (74) at the horizon $r^{2}=C^{2}$, i.e., $r_{*}=$ $-\infty$, simplifies to

$$
\left(\partial_{r_{*}}+2 i\left[\omega-\frac{L}{C^{2}} m\right]\right) \partial_{r_{*}} \phi_{\omega m}=0 .
$$

The two linearly independent solutions to this equation, i.e., (approximately) constant and purely oscillating, respectively, correspond to ingoing and outgoing modes, respectively.

In analogy to the Kerr metric we may introduce the angular velocity of horizon

$$
\Omega_{\mathrm{H}}=\frac{L}{C^{2}} .
$$

For a Kerr black hole this quantity is bounded by its total mass $M$ via $2 \Omega_{\mathrm{H}}<1 / M$. With the gravity wave analogs, however, it is possible to generate rather large values of $2 \Omega_{\mathrm{H}}$ - in particular if one allows for nonnegligible slopes - since $C$ and $L$ can be varied independently.

The Wronskian associated to the second-order ordinary differential equation reads at the horizon

$$
W_{\omega m}[\phi]=\phi^{*} \partial_{r_{*}} \phi-\phi \partial_{r_{*}} \phi^{*}+2 i\left(\omega-\Omega_{\mathrm{H}} m\right) \phi^{*} \phi .
$$

Inserting the ingoing mode $\partial_{r_{*}} \phi=0$ we obtain

\footnotetext{
${ }^{\ddagger}$ Otherwise the metric would describe a naked singularity without ergoregion and horizon, etc.
}

$$
W_{\omega m}[\phi]=2 i\left(\omega-\Omega_{\mathrm{H}} m\right)\left|\mathcal{T}_{\omega m}\right|^{2},
$$

where $\left|\mathcal{T}_{\omega m}\right|^{2}=\left|\phi_{\omega m}\right|^{2}$ denotes the transmission coefficient. Since we have restricted our solution to be purely ingoing at the horizon, it will contain ingoing as well as outgoing components (determined by the reflection coefficient $\mathcal{R}_{\omega m}$ ) at spatial infinity $r=\infty$ in general $\phi \propto \exp (-i \omega r)+\mathcal{R}_{\omega m} \exp (i \omega r)$. In this limit the wave equation (74) reduces to the usual form and hence the associated Wronskian reads

$$
W_{\omega m}[\phi]=2 i \omega\left(1-\left|\mathcal{R}_{\omega m}\right|^{2}\right) .
$$

For a regular second-order ordinary differential equation on a contiguous interval the Wronskian is conserved which implies [5] the following relation between the transmission $\mathcal{T}_{\omega m}$ and the reflection $\mathcal{R}_{\omega m}$ coefficients

$$
1-\left|\mathcal{R}_{\omega m}\right|^{2}=\frac{\omega-m \Omega_{\mathrm{H}}}{\omega}\left|\mathcal{T}_{\omega m}\right|^{2} .
$$

As one can easily infer from the equation above, if $m \Omega_{\mathrm{H}}>\omega$ the reflection coefficient is greater than one $\left|\mathcal{R}_{\omega m}\right|>1$, i.e. the scattered wave has a larger amplitude than the incident one. This amplification process corresponds to the phenomenon of superradiance.

However, for a massless scalar field in the asymptotically flat Kerr geometry the scattered wave escapes to infinity. To make an amplifier unstable, feedback is required, see 30]. That outgoing wave must be reflected back toward the hole for repeated amplification. This is possible if the field has a finite mass [3] or if one, for example, encloses the rotating black hole by a large spherical mirror [4].

As explained in Section IV, for the gravity wave analogs there is another mechanism which may force the scattered wave to "come back" - the total internal reflection. Since the velocity of the wave is $\sqrt{g h}$, if one can manipulate $h$, as happens for example near the drain of a bath tub, the change in the effective refractive index may entail effectively trapping the waves - which then can be amplified via superradiant scattering without bound, or, rather, until non-linear effects dominate.

In summary it is possible that some of the features which one regularly observes in the vortex flow near the drain of one's bathtub are in fact the result of instabilities which are exactly analogous to the behaviour of waves near a (rotating) black hole. It should be mentioned here that the arguments above do not explain the formation of the vortex, but refer to its linear instability.

\section{INNER HORIZON}

The instability indicated by negative parts of the energy density in Eq. (68) is not necessarily restricted to rotating black holes: In the presence of an inner horizon in addition to the outer one, such as occurring in the Reissner-Nordström metric, and for an altered dispersion 
relation, runaway solutions can exist even in the purely one dimensional flow.

This somewhat surprising fact has been demonstrated in Ref. [27]. In the following we give a brief repetition of the basic explanation adapted to subluminal high frequency dispersion relation. Note that the terms "sub-" or "superluminal" do not, despite their origin, refer to light, but rather to whether or not the group velocity at high wavenumbers is less than or greater than it is at very low wavenumber (long wavelength). They will also refer to regions where the velocity of the fluid is smaller or larger than than the low-wavenumber velocity of the waves.

We shall restrict ourselves to a one-dimensional flow. We consider a case where there are two horizons, one a white hole (where the velocity of the fluid drops below the low wavenumber velocity of the wave) and the other a black hole (where the velocity of the fluid goes above the low wavenumber group velocity). It turns out (for not entirely well understood reasons $\sqrt{(5)}$ that although there are two branches of the dispersion curve (for a static fluid these correspond to the two directions of propagation of the wave), wave packets remain on one or the other of these branches even when interacting with horizons, and spatially varying flows. There seems to be very little backscatter from one branch to the other. We shall restrict attention to the one branch which at low wavenumbers represents waves which are travelling in a direction opposite to the fluid flow.

Assuming adiabatic motion of this wave packet (in analogy to the geometric optics approximation), the effect of the fluid flow is to alter the dispersion relation to

$$
\omega+v k=F(k)
$$

where the mode has the form $e^{i(\omega t+k x)}$. I.e., $\omega$ is the frequency of the wave in the lab frame, not the rest frame of the fluid. Define $\Omega=\omega+v k$ as the frequency in the fluid rest frame. In Fig. 2 we sketch the subluminal dispersion relation as a plot of $\Omega$ versus $k$. To determine the possible values of $k$ for any given value of $\omega$, we can plot the line $\Omega-v k=\omega$ and look for the intercepts with the dispersion curve. We shall concentrate on small values of $\omega$, which is also the intercept of the line with the $\Omega$ axis.

On the subluminal side of the horizon, where $v$ and thus the slope of the line is less than the dispersion slope at $k=0$, there will in general be three points of intersection, and with very different group velocities. The one near the origin $k=0$ has a group velocity,

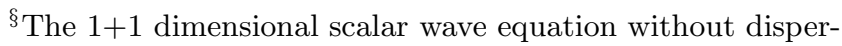
sion is conformally invariant which explains the absence of any mixing in this case. For a non-trivial dispersion, however, this problem is less clear.
}

$$
v_{\mathrm{g}}=-\frac{d \omega}{d k}=v-\frac{d F}{d k}
$$

which is negative, corresponding to travel to the left, against the flow of the current. The other two, one at positive $\Omega$ and one at negative $\Omega$ both have positive group velocity and thus correspond to packet travel with the current flow. (Their rest frame group velocity is so small that even the subluminal current is sufficiently fast to drag them along).

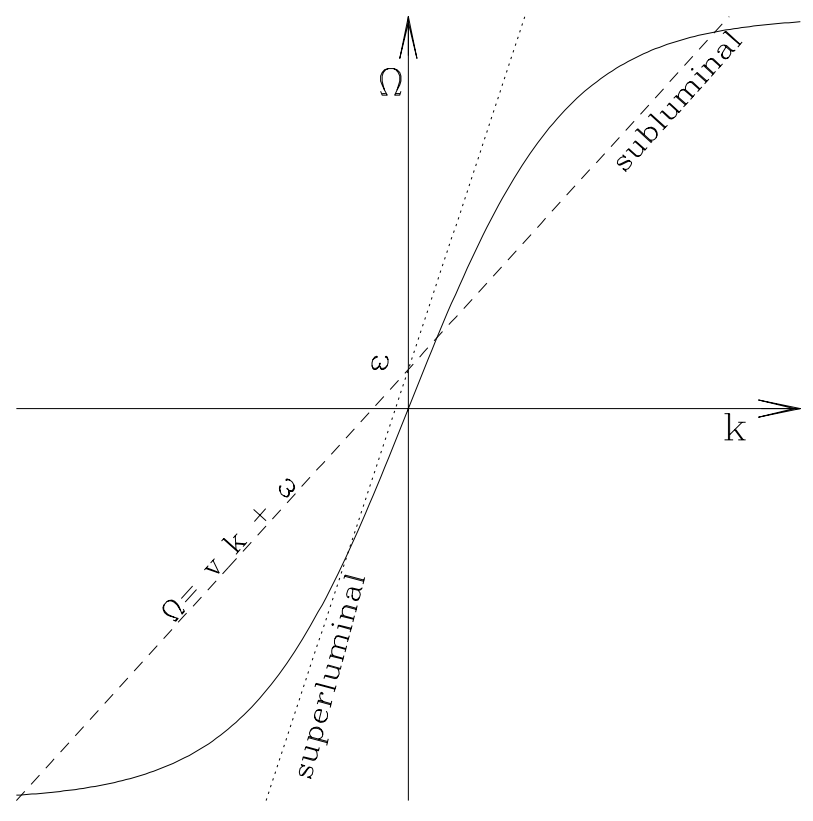

FIG. 2. One branch of the dispersion relation where the high wavenumber group velocity is smaller than the low frequency. Plotted are the two lines of $\Omega$ vs. $k$ for different velocities, but the same value of the lab frame frequency $\omega$. Note the intersection points of these straight lines with the dispersion curve indicating the possible values or $k$ for the given $\omega$ at the given fluid velocity.

Because the background current flow is assumed to be stationary, the frequency $\omega$ is conserved during the motion of a wave packet. Let us consider what the possible wavenumbers are for this same value of $\omega$ on the superluminal side. The slope of the line is now much greater than the low wavenumber slope of the dispersion curve, and there is now only one intersection with the dispersion curve, at negative values of $\Omega$. Furthermore the group velocity here is positive. These wave packets, even though they have low wavenumber, are dragged along with the fluid and travel in the same direction as the fluid.

One can, as for scalar waves, define a "norm" of the various wave packets by the conserved Klein-FockGordon inner product 


$$
\begin{aligned}
(\Phi \mid \Phi) & =\frac{i}{2} \int d \Sigma^{\mu}\left(\Phi^{*} \partial_{\mu} \Phi-\Phi \partial_{\mu} \Phi^{*}\right) \\
& =\frac{i}{2} \int d \Sigma^{\mu} \Phi^{*} \stackrel{\leftrightarrow}{\partial}_{\mu} \Phi \\
& =\frac{i}{2} \int d x \Phi^{*}\left(\stackrel{\leftrightarrow}{\partial}_{t}-v(x) \stackrel{\leftrightarrow}{\partial}_{x}\right) \Phi \\
& =\Omega(x) \int d x|\Phi|^{2},
\end{aligned}
$$

where we have assumed the wave packet to be sufficiently localized that $v$ is constant over the packet. I.e., the sign of the norm is the same as the sign of $\Omega$. Thus, for positive $\omega$ the wave packets on the superluminal side will have negative norm, while those on the subluminal side can be either positive or negative norm (two are positive and one is negative).

Let us consider a wave paket with positive norm, low value of $\omega$ but large value of $k$ being dragged toward the black hole horizon. As it comes closer, $v$ increases, and the wavelength of the packet is stretched out, with $k$ becoming smaller and smaller. As it hits the horizon, where the slope of the line becomes essentially the same as that of the low wavenumber dispersion curve, the adiabatic assumption (geometric optics approximation) fails, and one gets a mixing of the various possible values of $k$ for the given $\omega$. However, whatever happens, the wave packet must thereafter leave the horizon, either on the subluminal side, or the superluminal. On the subluminal side the only possibility is that the wave exit with the small value of $k$ near zero, as this is the only one of the three possibilities which travels away from the horizon on the subluminal side. On the superluminal side, the only possibility is that the wave packet leave with negative norm. The fluid flow at the horizon will have mixed positive with negative norm solutions. This is precisely the requirement in the quantum system that particle production take place, since this mixing of positive and negative norms is precisely what leads to non-trivial Bogoliubov coefficients

$$
\beta=\left(\Phi_{\text {in }}^{*} \mid \Phi_{\text {out }}\right) .
$$

For our purpose, what this means is that the wave packet which leaves the horizon on the subluminal side must have a larger norm than did the wave packet which was dragged toward the horizon. (Since the total norm is conserved, and since the norm of the packet on the superluminal side is negative, the subluminal packet must be larger.)

This packet will now travel toward the white hole horizon. Here, there is no packet on the superluminal side which travels away from the horizon. The only possibility is that the packet be blue shifted at the white hole horizon and come off as a mixture of the positive and negative large $k$ solutions. While one of these is positive norm, and the other is negative, they are travelling together toward the black hole horizon. Here they again emit a negative norm packet into the superluminal region, although if the phases were just right, the two could cancel and emit nothing into the superluminal side. Assuming that this does not happen, or that the conversion at the white hole is so small so as not to create any mixture, the subluminal packet's norm increases once again. After many such back and forth reflections, the norm can grow arbitrarily large. One has an instability. In Fig. 3 we have four steps in this process. The black hole horizon occurs in the center of the diagram, while the white hole horizon is at the left and right edges (we assume periodic coordinates). The low frequency intial wave packet travells toward the white hole horizon, and reflects as a high frequency wave, dragged by the fluid. The third window is after the reflection from the back hole, and the fourth after the refection again from the white hole.

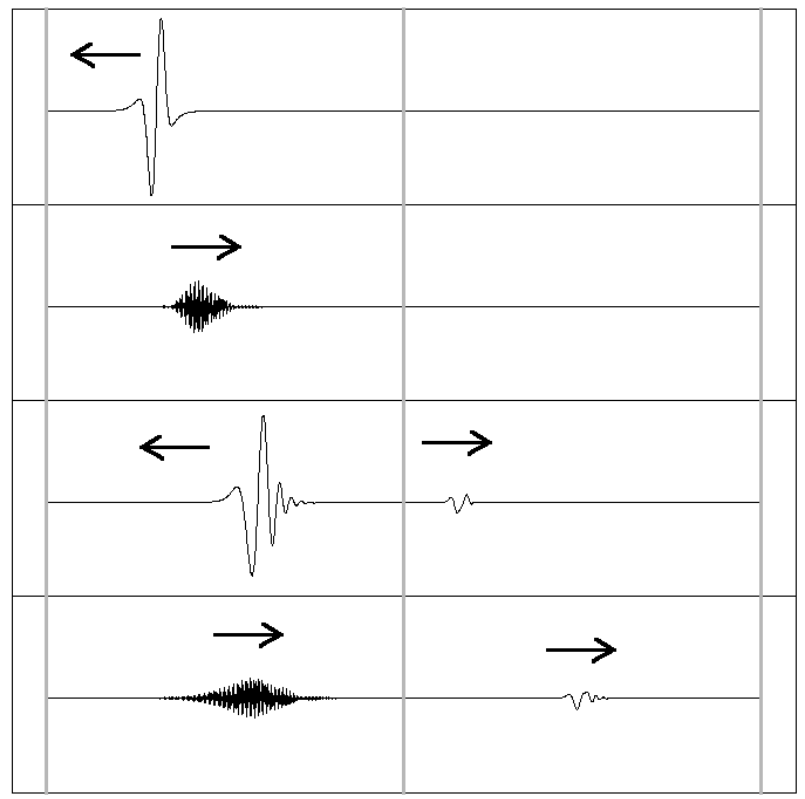

FIG. 3. Four stages in the process of a wave packet reflecting off the white and black hole horizons. The black hole horizon is in the centre. These are the real parts of the wave packets (the imaginary look similar and were chosen to make the intial pulse purely positive norm).

For small $\omega$ the amount of mixing of positive and negative norm modes at each of the horizons is governed by a thermal Bose-Einstein factor

$$
|\beta|^{2} \propto \frac{1}{\exp (\omega / T)-1},
$$

with an effective temperature $T$ proportional to the effective surface gravity, i.e., the rate of change of the velocity or fluid flow across the horizon. If one makes the white hole horizon have a very low effective temperature, one can minimize the creation of large $k$ negative norm solutions on "reflection" of the packet from this horizon. 
This means that the packet on the subluminal side will remain positive norm, if it started thus.

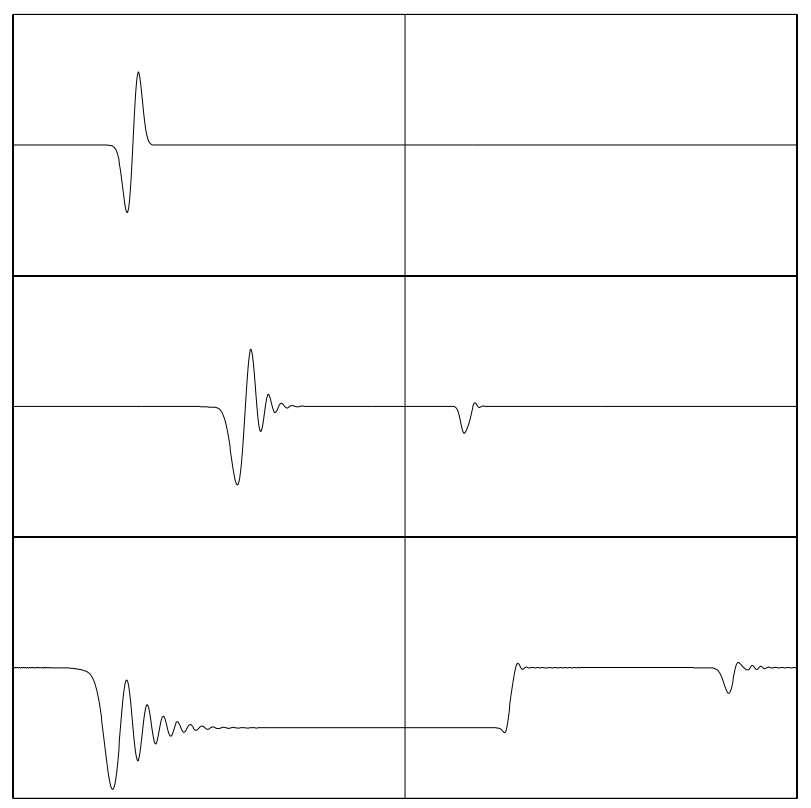

FIG. 4. The reflection of a pulse producing low frequency instability. Plotted are three steps in the evolution of a pulse (top box) after one (middle box) and two reflections from the black hole horizon in the center. Note that in each case the pulse on the left (in the subluminal region) is travelling to the left while the ones on the right (in the superluminal region) travel to the right. After another reflection from the black hole horizon, the pulse grows linearly and after still another, quadratically with distance from the black hole horizon.

One of the consequences of this instability is that one expects it to be worst for very small values of $\omega$. Since the norm mixing at the black hole horizon is proportional to a Bose-Einstein factor, this diverges for small values of $\omega$

$$
\frac{1}{\exp (\omega / T)-1} \stackrel{\omega \downarrow 0}{\longrightarrow} \frac{T}{\omega}
$$

One would thus expect, and numerical simulations confirm, that after a few back and forth bounces off the black hole horizon, the lowest wavenumber mode of the wave (i.e., constant in space) would be amplified at each bounce. Numerical simulation shows that after a couple of bounces, at each reflection from the black hole horizon, a constant "step function like" pulse of ever increasing amplitude is emitted by the black hole horizon into the subluminal side, cf. Fig. 4. After the next reflection the spatial groth is linear, then quadratic etc.

One can, following Corley and Jacobson [27, make a very similar analysis in the case in which the dispersion relation is superluminal

$$
F(k)=c^{2} \sqrt{k^{2}+a^{2} k^{4}},
$$

which is exactly the situation considered in Section V. In that case one should consider a superluminal region between a black hole and a white hole horizon. In this case it is the wave packet bouncing between these two horizons on the superluminal side which creates the instability. Taking positive $\omega$ modes but with an intial negative norm wavepacket, we find that every time the packet hits the black hole horizon it emits a positive norm packet into the subluminal region beyond that horizon (which is again exactly the classical counterpart of Hawking radiation), thus increasing the size of the negative norm packet in the superluminal region, while every time it bounces off the white hole horizon, it simply bounces off, possibly mixing in some high frequency positive norm packet as well (depending again on the effective temperature of the horizon).

Since with gravity waves, we can choose whether to have a subluminal or superluminal dispersion relation, by choosing the depth and surface tension of the liquid, one can test both sets of predictions. Furthermore, since this norm mixing property of the horizon is intimately related to the quantum emission of radiation by the horizon, one can, with purely classical gravity waves, test the predictions about the thermal behaviour of the various types of horizon.

The transition between short and long gravity waves can be described by the height-dependent dispersion relation for a fluid at rest 26]

$\omega^{2}=g k \tanh \left(k h_{\mathrm{B}}\right)=g h_{\mathrm{B}} k^{2}\left(1-\frac{h_{\mathrm{B}}^{2}}{3} k^{2}\right)+\mathcal{O}\left(k^{6}\right)$.

This should be contrasted with the dispersion of the surface tension waves as derived in Eq. (54) of Section $\mathrm{V}$ (again for a fluid at rest)

$$
\omega^{2}=g h_{\mathrm{B}} k^{2}\left(1+a^{2} k^{2}\right) .
$$

Consequently, one obtains a superluminal dispersion relation for $a \approx h_{\mathrm{B}}$ which implies $h_{\mathrm{B}} \approx 2 \mathrm{~mm}$ for mercury. For $a \ll h_{\mathrm{B}}$, on the other hand, the dispersion relation possesses a large subluminal region (short gravity waves) before it becomes superluminal (surface tension waves) again.

A possible experimental set-up for observing this instability is sketched in Fig. 5. The fluid is flowing within a circular basin with varying depth such that the liquid's flow velocity as well as the speed of the gravity waves depends on the position. As the depth of the basin decreases the velocity of the fluid increases and in the same time the speed of the gravity waves decreases.

In this way one may construct a region of superluminal fluid velocity confined between the inner and the outer horizon. As mentioned above, the gradient of the basin's depth (determining the the surface gravity) should be small at the inner (white hole) horizon whereas it should 
be as large as possible - without violating the assumption in Section [I], e.g. by generating a breakdown of the laminar flow - at the outer (black hole) horizon in order to observe the instability described above.

On the other hand, one also has to make sure that the wave packets emitted by mode conversion into the other region do not travel around and disturb the system when they come back through the other horizon. For real black holes they escape to infinity. In our system, however, this is not the case and thus one has to install an additional absorbing device, which effectively models spatial infinity - in order to prevent this interference.

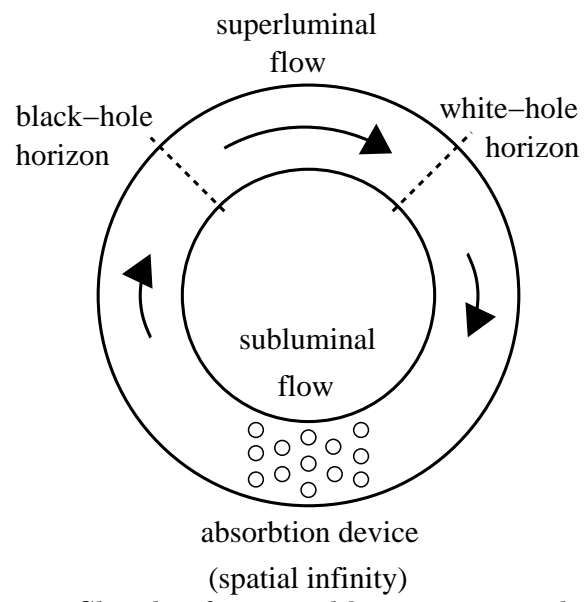

FIG. 5. Sketch of a possible experimental set-up. A wavepacket is bouncing back and forth in the superluminal region between the inner (white hole) and the outer (black hole) horizon, emitting a piece of "Hawking radiation" at the black hole horizon and thereby increasing its amplitude.

According to the remarks in Section V1 it appears to be reasonable to move the bottom (e.g. made of rubber) of the fluid or exert a magnetic force on it. In practice one might generate a flow with a slowly increasing velocity until the two horizons are formed. In this case one should observe an instability of the laminar and smooth flow profile exactly at this threshold velocity. Of course one has to ensure that there is no other instability at or before this thershold which might spoil the experiment.

\section{DISCUSSION}

In view of the main advantage of the gravity wave analogs - the possibility of tuning the velocity of the wave propagation rather independently - one would expect that it is possible to simulate black and white holes in the laboratory and to study their instabilities experimentally. As one perhaps reasonable hierarchy of the different dimensions involved $\delta h \ll h_{\mathrm{B}} \ll \lambda$ one could imagine waves of about $1 \mathrm{~mm}$ amplitude in a $1 \mathrm{~cm}$ deep basin with the wavelength being circa $10 \mathrm{~cm}$ and the characteristic size of the black or white hole analog (its Schwarzschild radius) approximately $1 \mathrm{~m}$. The velocity of those waves - and hence also that of the fluid - in this case would be about $0.3 \mathrm{~ms}^{-1}$ and should be realizable. Aiming for the incorporation of surface tension waves for large wavenumbers it might be suitable to divide the above suggested length scales by a factor of about 10 . Here we encounter another advantage of the gravity wave analogs: In contrast to sound waves, for example, the amplitude of the gravity waves can be measured directly (as a length) and with a very high accuracy (e.g. via interferometry using the reflection at the fluid's surface).

However, all the suggestions above have to be reconsidered when aiming for an experimental verification of the Hawking effect [11]. In order to detect this quantum instability the temperature of the fluid should be as small as possible - whereas the fluid's velocity and its gradient have to assume their maximal feasible values. The experimental verification of the Hawking effect seems to be on or even still beyond [25] the edge of our present experimental capabilities - for the dielectric/optical as well as for the sonic/acoustic black hole analogs (Dumb holes) and probably even more so for the gravity wave analogs.

\section{ACKNOWLEDGEMENT}

This work was supported by the Alexander von Humboldt foundation, the Canadian Institute for Advanced Research, and the NSERC.

[1] C. M. Misner, K. S. Thorne, and J. A. Wheeler, Gravitation, (Freemann, San Francisco, 1973);

[2] M. A. Abramowicz and A. R. Prasanna, Mon. Not. R. astr. Soc. 245, 720 (1990); see also A. Y. Shiekh, Centri'fugal' force around a black hole, e-preprint: astro-ph/9211005 and references therein.

[3] S. Detweiler, Phys. Rev. D 22, 2323 (1980); T. J. Zouros and D. M. Eardley, Annals Phys. 118, 139 (1979); T. Damour, N. Deruelle, and R. Ruffini, Lett. Nuovo Cim. 15, 257 (1976).

[4] W. H. Press and S. A. Teukolsky, Nature 238, 211 (1972).

[5] R. Penrose, Nuovo Cimento 1 (special number), 252 (1969); Y. B. Zel'dovich, Pisma Zh. Éksp. Teor. Fiz. 14, 270 (1971); JETP Lett. 14, 180 (1971); Zh. Éksp. Teor. Fiz. 62, 2076 (1972); Sov. Phys. JETP 35, 1085 (1972); A. A. Starobinski, Sov. Phys. JETP 37, 28 (1973); B. S. DeWitt, Phys. Rept. 19, 295 (1975); J. D. Bekenstein and M. Schiffer, Phys. Rev. D 58, 064014 (1998); N. Andersson, P. Laguna, and P. Papadopoulos, ibid. 58, 087503 (1998).

[6] D. M. Eardley, Phys. Rev. Lett. 33, 442 (1974).

[7] Y. B. Zel'dovich, I. D. Novikov, and A. A. Starobinski, Zh. Éksp. Teor. Fiz. 66, 1897 (1974); Sov. Phys. JETP 39, 933 (1974). 
[8] R. M. Wald and S. Ramaswamy, Phys. Rev. D 21, 2736 (1980).

[9] R. Schützhold, Phys. Rev. D 64, 024029 (2001).

[10] M. Simpson and R. Penrose, Int. J. Theor. Phys. 7, 183 (1973); E. Poisson and W. Israel, Phys. Rev. Lett. 63, 1663 (1989); Phys. Rev. D 41, 1796 (1990).

[11] S. W. Hawking, Nature 248, 30 (1974); Commun. Math. Phys. 43, 199 (1975).

[12] J. D. Bekenstein, Lett. Nuovo Cim. 4, 737 (1972); Phys. Rev. D 7, 2333 (1973); ibid. 9, 3292 (1974); ibid. 12, 3077 (1975).

[13] S. Dimopoulos and G. Landsberg, Phys. Rev. Lett. 87, 161602 (2001).

[14] W. G. Unruh, Phys. Rev. Lett. 46, 1351 (1981).

[15] M. Visser, Class. Quant. Grav. 15, 1767 (1998); S. Liberati, S. Sonego, and M. Visser, Class. Quant. Grav. 17, 2903 (2000).

[16] G. E. Volovik, Phys. Rept. 351, 195 (2001); Pisma Zh. Éksp. Teor. Fiz. 69, 662 (1999); JETP Lett. 69, 705 (1999); U. R. Fischer and G. E. Volovik, Int. J. Mod. Phys. D 10, 57 (2001); G. E. Volovik, Universe in a Helium Droplet, to be published in Oxford University Press.

[17] L. J. Garay et al, Phys. Rev. Lett. 85, 4643 (2000); C. Barcelo, S. Liberati, and M. Visser, Class. Quant. Grav. 18, 1137 (2001).

[18] C. Barcelo, M. Visser, and S. Liberati, Int. J. Mod. Phys. D 10, 799 (2001).

[19] M. Visser, Phys. Rev. Lett. 80, 3436 (1998).

[20] H. C. Rosu, Grav. Cosmol. 7, 1 (2001); Int. J. Mod. Phys. D 3, 545 (1994); C. Barcelo, S. Liberati, and M. Visser, Class. Quant. Grav. 18, 3595 (2001).

[21] M. Novello and J. M. Salim, Phys. Rev. D 63, 083511 (2001); F. Baldovin et al, Class. Quant. Grav. 17, 3265 (2000); M. Novello et al, Phys. Rev. D 61, 045001 (2000).

[22] U. Leonhardt and P. Piwnicki, Phys. Rev. Lett. 84, 822 (2000); see also M. Visser, ibid. 85, 5252 (2000); U. Leonhardt and P. Piwnicki, ibid. 85, 5253 (2000).

[23] B. Reznik, Phys. Rev. D 62, 044044 (2000).

[24] R. Schützhold, G. Plunien, and G. Soff, Phys. Rev. Lett. 88, 061101 (2002).

[25] W. G. Unruh, Measurability of Dumb Hole Radiation?, to appear in Artificial Black Holes, edited by Mario Novello, Matt Visser, and Grigori Volovik.

[26] L. D. Landau and E. M. Lifshitz, Fluid Mechanics, Volume 6, Course of Theoretical Physics (Pergamon, London, 1959).

[27] S. Corley and T. Jacobson, Phys. Rev. D 59, 124011 (1999).

[28] M. Stone, Phys. Rev. E 62, 1341 (2000).

[29] P. Painlevé, C. R. Hebd. Seances Acad. Sci. (Paris) 173, 677 (1921); A. Gullstrand, Ark. Mat. Astron. Fys. 16, 1 (1922); G. Lemaître, Ann. Soc. Sci. (Bruxelles) A 53, 51 (1933).

[30] H. R. Beyer, Commun. Math. Phys. 221, 659 (2001); B. F. Whiting, J. Math. Phys. 30, 1301 (1989). 